(C) Elsevier/INRA

Article original

\title{
Description de la diversité génétique chez Brassica oleracea à l'aide de marqueurs isoenzymatiques et moléculaires
}

\author{
E Margalé, AM Chèvre, R Delourme, Y Hervé \\ Institut national de la recherche agronomique, \\ station de génétique et d'amélioration des plantes, \\ 35650 Le Rheu, France
}

\begin{abstract}
Résumé - Deux méthodes d'analyse de la diversité génétique sont comparées dans le but de constituer des pools génétiques homogènes en vue de la multiplication d'une collection de choux (Brassica oleracea). Il apparaît que la variabilité génétique intrapopulation est facilement accessible par la méthode isoenzymatique. Cependant, pour la structuration des populations, c'est la méthode RAPD (amplification aléatoire d'ADN) qui fournit les informations les mieux corrélées avec la diversité morpho-agronomique et pour un moindre coût. La méthode proposée repose sur l'analyse de la présence-absence d'une centaine de marqueurs RAPD par échantillon, lui-même constitué de l'ADN en mélange de 40 graines. L'estimation des distances inter-populations et inter-cultigroupes par cette technique rend plus aisée la structuration de la collection. Les données permettent la construction d'arbres phylogénétiques à l'aide du logiciel PAUP (Phylogenetic Analysis Using Parsimony).
\end{abstract}

Brassica oleracea / diversité / collection / isoenzyme / RAPD

Summary - Description of genetic diversity within Brassica oleracea using isoenzymatic and molecular markers. The aim of this work is to construct homogeneous genetic pools for the multiplication of a crop collection of Brassica oleracea. Two methods of analysis of genetic diversity were compared. For intra-population investigations, the isoenzymatic method is more efficient. However, for studying population structure, the information given by RAPD (randomly amplified DNA sequence) is better correlated with morphological and agronomical diversity and is less expensive. The suggested method involves testing for the presence or absence of about 100 RAPD markers in a sample of mixed DNA from 40 seeds. The appropriate structure for the collection can be determined from inter-population and inter-cultigroup distances estimated by this technique. The data allow the construction of phylogenetic trees by the PAUP software (phylogenetic analysis using parsimony).

Brassica oleracea / diversity / collection / isozyme / RAPD 


\section{INTRODUCTION}

Les méthodes actuelles d'évaluation de la diversité génétique sont nombreuses (Lefort-Buson et de Vienne, 1985). Cependant la presque totalité des collections est évaluée au champ à l'aide de caractères phénotypiques (morphologiques, biométriques, agronomiques) soumis à la sélection. La collecte des résultats est bien souvent très coûteuse en main d'œuvre et en temps. D'autres techniques permettent d'évaluer la diversité des caractères réputés neutres vis-à-vis de la sélection naturelle. Citons par exemple, l'analyse isoenzymatique, l'analyse protéique monoou bidimensionnelle, l'analyse RFLP (polymorphisme de longueur de fragment de restriction), ou plus récemment l'analyse RAPD (amplification aléatoire d'ADN).

Ce travail concerne l'évaluation de la diversité génétique d'une collection de choux cultivés (Brassica oleracea L), rassemblée en 1983. Le chou est une espèce très polymorphe qui a été domestiquée par les Celtes pendant le premier millénaire avant JC (Prakash et Hinata, 1980). Depuis, de nombreuses variétés botaniques (tableau I) (Crisp, 1975) ont été sélectionnées pour permettre la consommation de tous les organes aériens de la plante : l'hypocotyle pour le chou-rave, la tige et les feuilles pour le chou fourrager, les bourgeons axillaires pour le chou de Bruxelles, le bourgeon terminal pour le chou-pommé, la pré-inflorescence pour le chou-fleur et l'inflorescence pour le chou brocoli.

La collection regroupe 1100 populations, parmi lesquelles de nombreuses populations fermières de choux-fleurs (convar botrytis), de choux pommés (convar capitata) et de choux fourragers (convar acephala). Après récolte, les graines ont été stockées dans un congélateur à $-18^{\circ} \mathrm{C}$. Cette conservation n'ayant pas reçu de soins particuliers (contrôle de l'humidité) et la qualité des stocks initiaux étant parfois médiocre, le taux de germination des graines a subi une diminution importante allant même pour certains lots jusqu'à des valeurs inférieures à $5 \%$. Dans ces conditions, une multiplication était urgente pour assurer la pérennité de la collection.

La multiplication de 1100 populations de cette espèce préférentiellement allogame (excepté pour les choux-fleurs d'été) nécessite donc la prévention de toute pollinisation étrangère (isolements, cages de multiplication) et la réduction du nombre de lots à multiplier, soit en faisant un choix arbitraire de cultivars, soit en créant des pools de populations. C'est cette dernière option qui a été retenue.

Pour ce faire, nous avons analysé la diversité génétique d'un échantillon des populations précédentes à partir de marqueurs isoenzymatiques et RAPDs. La comparaison des résultats obtenus avec ces deux types de marqueurs et leur confrontation avec ceux obtenus pour quelques caractères morphologiques et agronomiques, constitue l'essentiel de l'article. Elle conduit à discuter la pertinence des informations moléculaires à recueillir pour la constitution de pools génétiques.

\section{MATÉRIELS ET MÉTHODES}

\section{Matériels}

Les populations collectées sont des cultivars locaux améliorés par les producteurs. Nombre d'entre eux ont plus d'un siècle de sélection et les semences ont été jalousement transmises de génération en génération de maraîchers. Les caractéristiques 


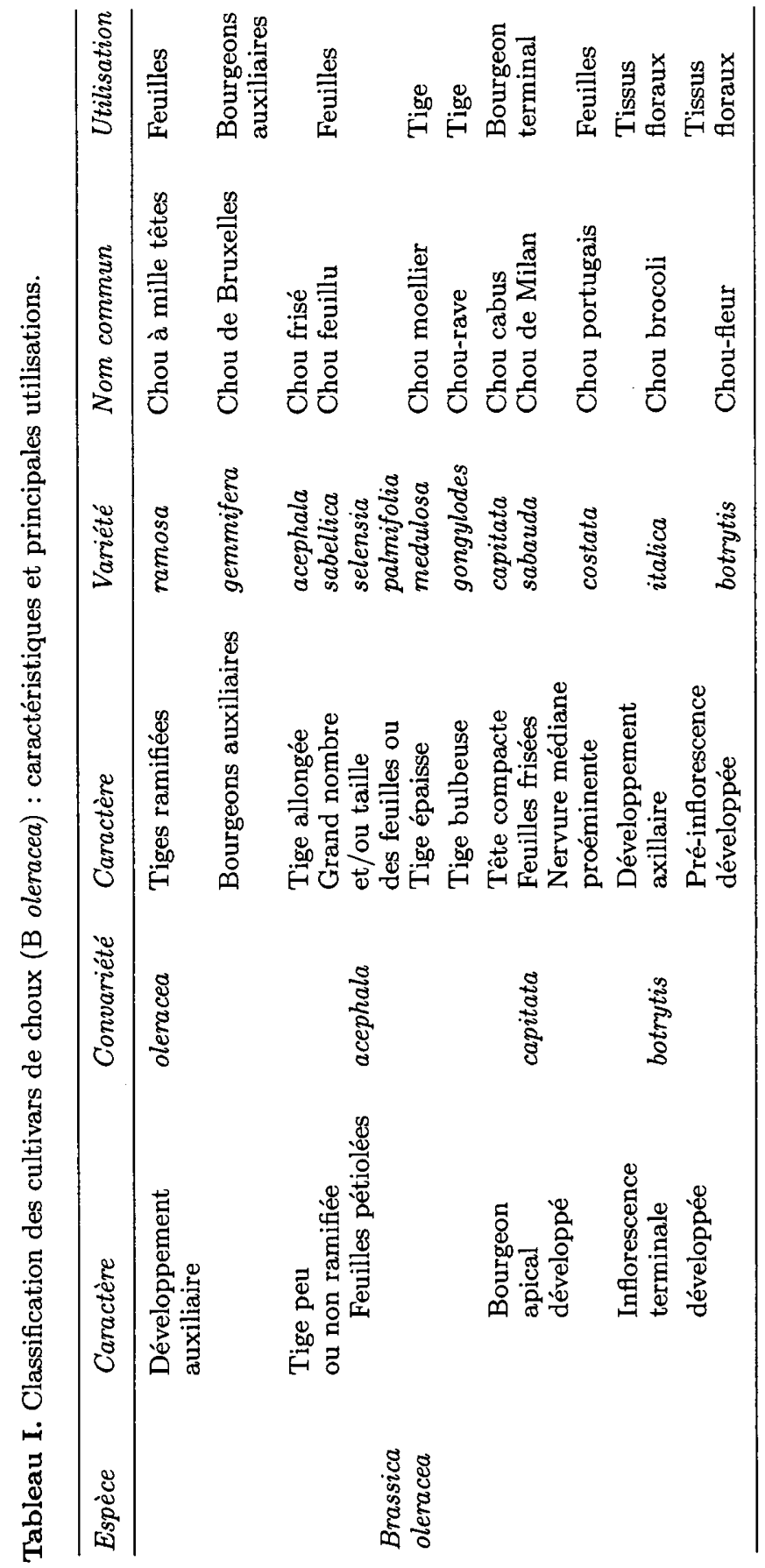


des cultivars compilées dans un répertoire font état de la précocité, du type morphologique approximatif (défini selon des critères propres à chaque producteur), du lieu de production et éventuellement de caractères spécifiques. Peu de données ont été communiquées sur les méthodes de sélection de ces populations. Cependant, nous savons que la production de graines était réalisée sur quelques plantes «portegraines» sélectionnées dans les parcelles de culture puis regroupées en isolements pour la production de semences. Cette production n'était pas systématiquement reconduite tous les ans mais le plus souvent tous les 2 à 3 ans (Hervé, communication personnelle).

L'échantillon de populations étudiées a été choisi parmi les 3 principaux cultigroupes : les choux-fleurs, les choux pommés et les choux fourragers. Chaque population est codée par un groupe de lettres (FLE et FLH pour choux-fleurs d'été et d'hiver respectivement; PO pour choux pommés et FO pour choux fourragers), suivi de 2 nombres (le code du département d'origine et le numéro d'entrée dans la collection). Par ailleurs, les populations Protéor et Flamcaul sont des variétés de choux fourragers cultivées, M-71 et DJ 105b de choux-fleurs, de précocité de mai et décembre-janvier respectivement.

L'analyse de la diversité isoenzymatique porte sur 43 populations de choux (11 choux-fleurs, 10 choux pommés et 22 choux fourragers) représentées chacune par un effectif de 30 plantes.

La comparaison des résultats acquis par les marqueurs isoenzymatiques et RAPDs est réalisée au niveau individuel sur 48 plantes appartenant à 11 populations de choux et issues des 3 cultigroupes précités. L'effectif pour chaque population varie de 3 à 7 individus.

La comparaison des arbres phylogénétiques obtenus à partir des 2 méthodes d'analyse a été réalisée sur un échantillon de 28 populations. Pour l'analyse isoenzymatique, cet échantillon porte sur un effectif de 30 plantes par population. Pour l'analyse RAPD, un échantillon unique de 40 graines en mélange est utilisé pour chaque population.

Le tableau II résume les caractéristiques des 3 études réalisées dans ce travail.

\section{Méthodes d'analyse de la diversité génétique}

\section{L'analyse isoenzymatique}

Les 6 systèmes isoenzymatiques retenus dans cette étude sont ceux pour lesquels le déterminisme génétique est au moins partiellement connu. Les 10 gènes présentant de la variabilité dans cette analyse sont les suivants : Lap-1, Pgi-2, Aco-2, Aco-3, Aco-4, Got-3, 6Pgd-1, 6Pgd-2, Pgm-2, Pgm-3. Les méthodes d'électrophorèse et de révélation utilisées ont été décrites dans une précédente communication (Arùs $e t$ al, 1991).

\section{L'analyse RAPD}

Cette technique, basée sur l'amplification de séquences d'ADN à partir d'amorces de 10 acides nucléiques (Opéron Technologies Society) et de Taq polymérase (Promega) permet de séparer sur un gel d'agarose les séquences amplifiées (Williams 


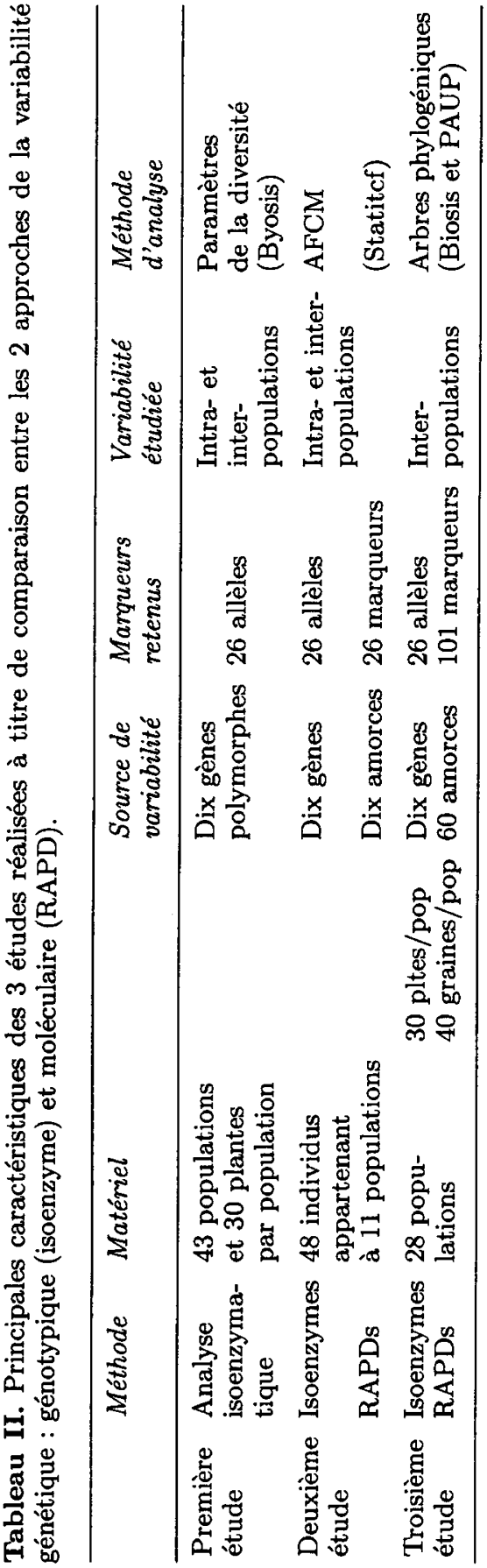


et al, 1990). L'extraction de l'ADN est faite sur feuilles pour l'analyse plante à plante et à partir d'un mélange de graines pour l'analyse comparative des populations. La méthode utilisée est celle décrite par Dellaporta et al (1983) pour l'extraction sur feuilles et par une méthode Dellaporta modifiée et adaptée à l'extraction de l'ADN de graines (Margalé, soumis). L'amplification est réalisée avec un Thermal cycler de la société Perkin Elmer Cetus programmé pour 45 cycles à $92^{\circ} \mathrm{C}$ pendant 1 min, à $35^{\circ} \mathrm{C}$ pendant $1 \mathrm{~min}$, et à $72^{\circ} \mathrm{C}$ pendant $2 \mathrm{~min}$ pour la dénaturation, l'hybridation et l'extension de la chaine d'ADN respectivement. Nous avons choisi pour marqueur toute bande nette, de taille distincte des autres bandes, et polymorphe.

\section{Méthodes d'analyse des résultats}

Pour la première expérience, essentiellement concernée par des résultats isoenzymatiques, tous les paramètres de la diversité génétique sont analysés à l'aide du logiciel Biosys développé par DL Swofford.

Pour la seconde expérience, nous avons procédé à une analyse identique pour les 2 sources d'informations en considérant tous les marqueurs RAPDs et tous les allèles isoenzymatiques comme des caractères indépendants. Il est en effet admis que les marqueurs RAPDs sont, dans une large mesure, indépendants même si certains d'entre eux peuvent être liés. Pour l'analyse isoenzymatique, l'indépendance des gènes a été vérifiée par Arus et Shields (1983). Par ailleurs, la présence d'un allèle à un locus (même biallélique) ne conditionne pas la présence ou l'absence de l'autre allèle au niveau individuel. La présence d'un marqueur RAPD ou d'un allèle isoenzymatique est alors notée 1 et l'absence 0 dans une matrice de résultats binaire. Une analyse factorielle des correspondances multiples (AFCM) a ensuite été construite sur 5 axes.

Enfin, la construction des arbres phylogénétiques a été réalisée à partir des fréquences alléliques pour les marqueurs enzymatiques et des présences-absences des séquences amplifiées pour les RAPDs. Ces données ont été traitées avec la méthode dite de parcimonie de Wagner (Fitch et Margoliash, 1967), en utilisant les logiciels Biosys et PAUP développés par DL Swofford (logiciels non publiés).

\section{RÉSULTATS}

\section{Analyse de la diversité enzymatique globale}

Parmi les 6 systèmes isoenzymatiques retenus pour l'analyse, 10 gènes polymorphes ont révélé 26 allèles pour l'ensemble des individus. Le nombre d'allèles par gène varie de 2 à 4 . La quasi-totalité des allèles est présente dans les 3 cultigroupes, par contre leurs fréquences respectives sont variables. Le nombre moyen d'allèles par locus varie de 1 à 2,5 . Chez les choux-fleurs d'été, la moyenne est de 1,1 indiquant que presque tous les locus isoenzymatiques sont fixés pour le même allèle. C'est pour les choux fourragers que la moyenne est la plus forte avec des valeurs allant de 2 à 2,5 allèles par locus, et de 50 à $90 \%$ de locus polymorphes pour ces populations.

L'hétérozygotie moyenne varie de 0 pour certaines populations de choux-fleurs d'été à 0,438 pour certaines variétés de choux fourragers. Les écarts entre les hétérozygoties observée et attendue sont faibles et laissent supposer des conditions 
relativement proches de la panmixie pour la plupart des populations. Une analyse plus détaillée pour chaque population à chaque locus indique que des écarts significatifs par le test de $\chi^{2}$ ont été observés dans moins de $6 \%$ des situations. La moitié des populations ne présente aucun écart à aucun locus, et $95 \%$ des populations présentent au moins 10 locus sur 11 en équilibre. Aucun écart à la panmixie n'est observé pour les choux-fleurs d'été. Pour les choux-fleurs d'hiver et les choux fourragers, la présence d'un déséquilibre significatif est généralement associée à un déficit en hétérozygotes. Le résultat inverse est trouvé pour les choux pommés (tableau III).

L'organisation de la variabilité a été étudiée à partir des index de fixation de Wright intrapopulation (Fis), inter-populations (Fst), et totaux (Fit), estimés pour chaque locus. La valeur moyenne du Fis est cohérente avec le faible écart obtenu entre hétérozygoties théorique et observée. On note quelques écarts à cette moyenne pour certains gènes, avec un excès d'homozygotes pour le gène $P g m-3$ et un déficit pour les gènes $\mathrm{Aco}-4$ et $\mathrm{Pgi}-2$. Pour les gènes $\mathrm{Got-3}, \mathrm{Pgm}-2$ et $P g m-3$, les valeurs du Fit et du Fst révèlent une hétérozygotie élevée associée à une différenciation relativement importante entre les populations (tableau IV).

Les indices de fixation des locus polymorphes ont été décomposés pour chaque niveau de hiérarchie (convariété, variété botanique, origine géographique et population). Les résultats montrent des différences importantes entre les locus étudiés (tableau V). Le locus 6Pgd-1 présente des indices faibles et équivalents à tous les niveaux de hiérarchie, ce qui indique la nature aléatoire de ses fluctuations alléliques. Pour les locus Aco-4, Lap-1, 6Pgd-2, on note des variations de fixation uniquement entre les populations. Pour les locus Aco-2, Pgi-2, Pgm-2, Pgm-3, une différenciation est observée aux niveaux population, origine et variété. Une différenciation au niveau convariétal n'existe que pour Pgm-2, Aco-2 et Got-3, ce dernier locus révélant une différenciation à tous les niveaux. En effet, pour ce gène la fréquence des 2 allèles (c1 et $c 2$ ) est à peu près identique chez les choux pommés $\left(f_{c 1}=0,496\right.$ et $\left.f_{c 2}=0,504\right)$ alors qu'elle tend vers la fixation de $c_{1}\left(f_{c 1}=0,786\right)$ pour les choux-fleurs et vers la fixation de $c_{2}\left(f_{c 2}=0,789\right)$ chez les choux fourragers.

La matrice des distances génétiques indique une relative proximité des populations appartenant au même cultigroupe. Les valeurs des distances de Rogers (1972) ont été indiquées pour les convariétés (tableau VI). À l'intérieur des cultigroupes ce sont les choux pommés qui présentent les distances génétiques les plus grandes en raison des différences importantes entre les types Milan et cabus. Entre les cultigroupes, le type chou-fleur est le plus éloigné des autres et en particulier du chou fourrager.

\section{Comparaison de la diversité décrite avec 2 types de marqueurs}

Chacun des 48 individus est défini par 2 séquences binaires indiquant la présence ou l'absence de chacun des marqueurs isoenzymatiques et RAPDs. Les marqueurs isoenzymatiques utilisés sont les 26 allèles révélés sur l'ensemble de l'échantillon; pour l'analyse RAPD 26 marqueurs ont été révélés à partir des oligomères 1 à 10 du kit A. L'AFCM réalisée à partir de données isoenzymatiques a permis d'obtenir dans le premier plan, une séparation relative des choux-fleurs par rapport aux 


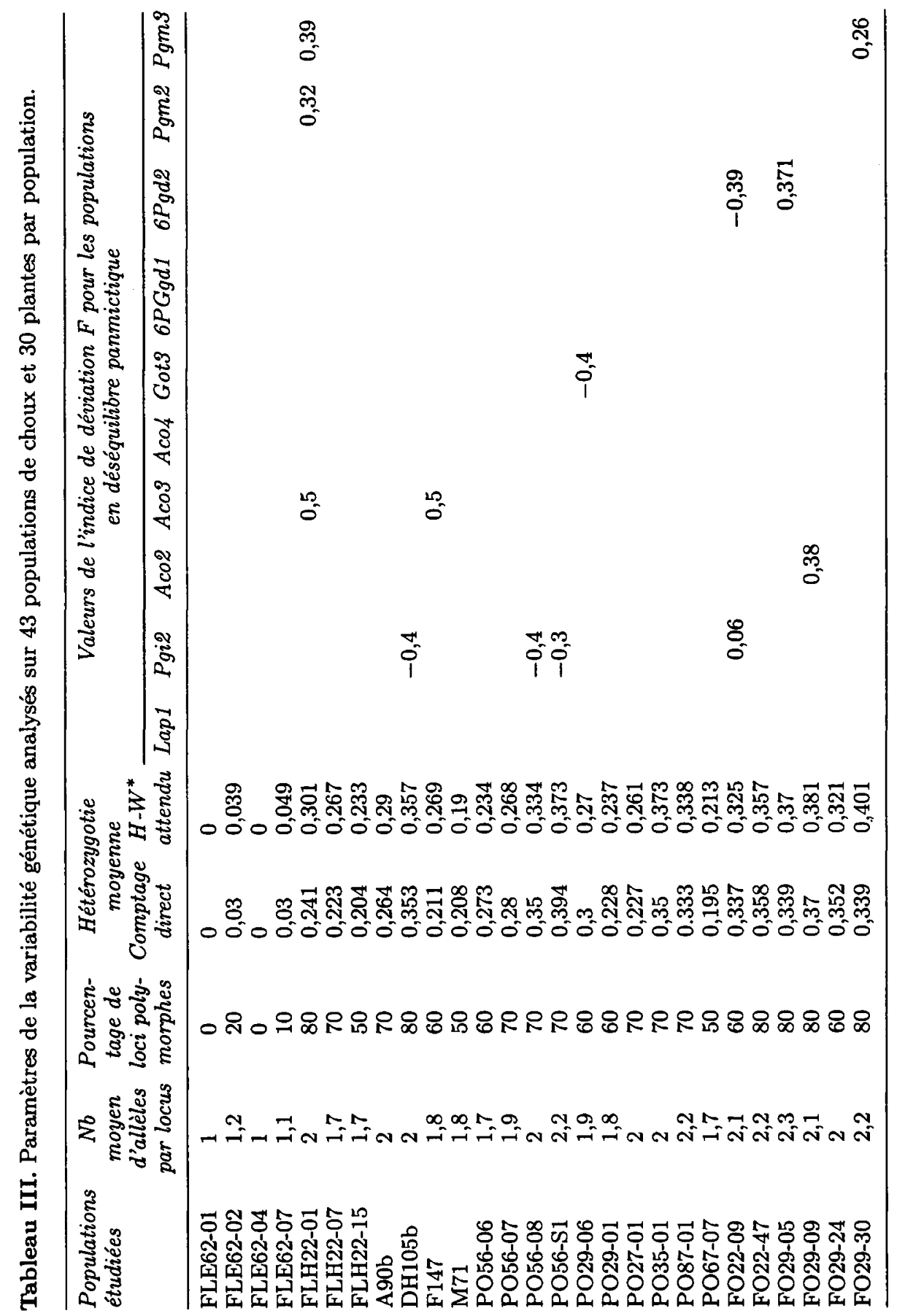


Diversité génétique du chou

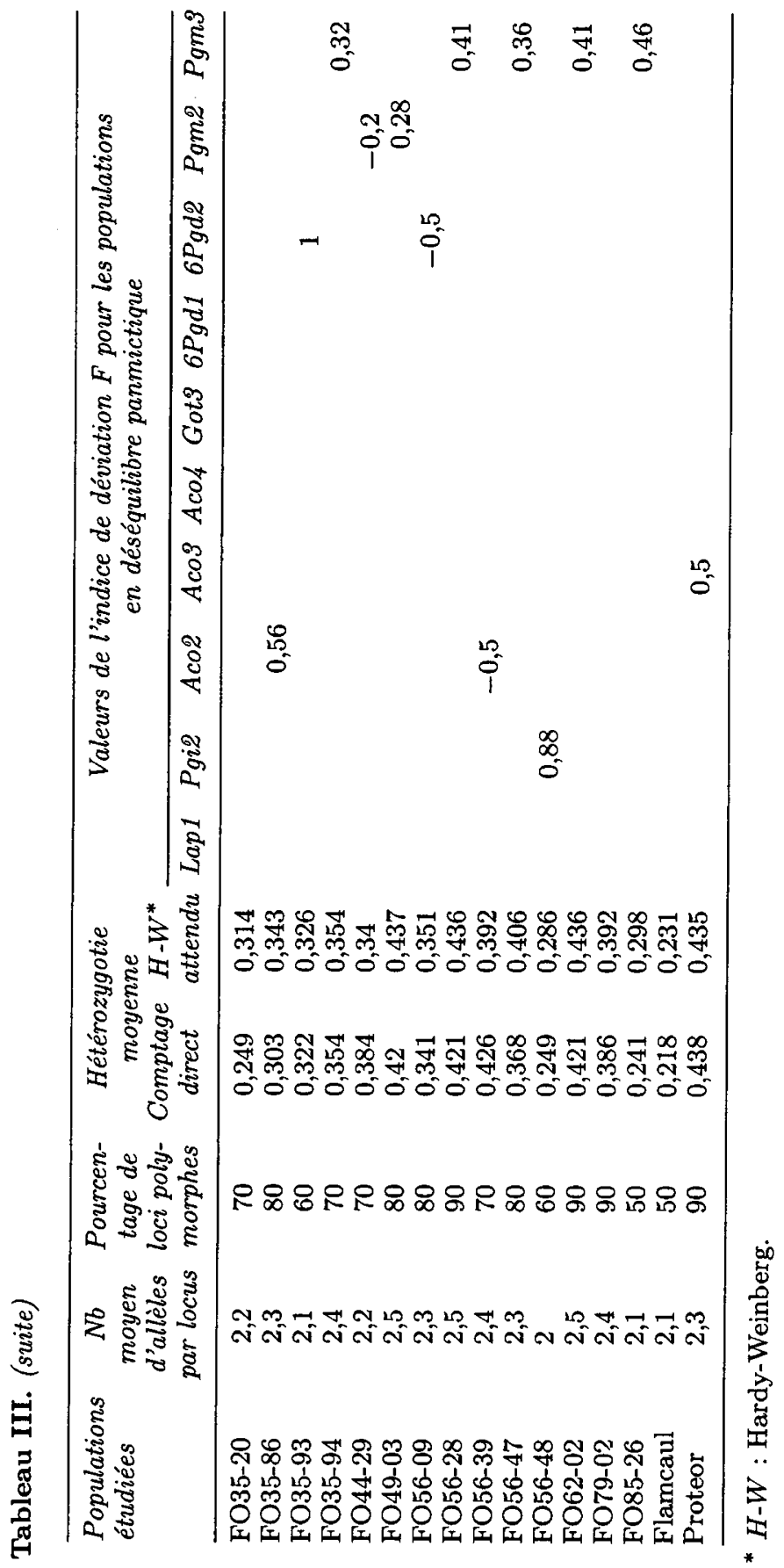


Tableau IV. Décomposition de l'indice de fixation de Wright intrapopulation (Fis), interpopulation (Fit) et total (Fst) pour chaque locus. La contribution à la différenciation entre les populations est principalement apportée par les gènes Got3, Pgm3 et Pgm2.

\begin{tabular}{lccl}
\hline Locus & $F(I S)$ & $F(I T)$ & $F(S T)$ \\
\hline Lap1 & $-0,059$ & 0,288 & 0,328 \\
Pgi2 & $-0,118$ & 0,154 & 0,243 \\
Aco2 & $-0,045$ & 0,305 & 0,335 \\
Aco3 & 0,069 & 0,352 & 0,304 \\
Aco4 & $-0,144$ & 0,241 & 0,336 \\
Got3 & $-0,005$ & 0,506 & 0,506 \\
6Pgd1 & $-0,072$ & $-0,005$ & 0,062 \\
6Pgd2 & 0,01 & 0,278 & 0,271 \\
Pgm2 & 0,04 & 0,419 & 0,394 \\
Pgm3 & 0,167 & 0,494 & 0,393 \\
Moyennes & $-0,006$ & 0,035 & 0,35 \\
\hline
\end{tabular}

autres choux. Pour les types pommés et fourragers, la distinction est beaucoup moins nette (fig $1 \mathrm{~A}$ ). Les résultats de l'analyse RAPD indiquent aussi une très nette distinction des plantes appartenant au cultigroupe chou-fleur suivant l'axe 1 de l'AFCM. L'axe 2 quant à lui distingue très nettement les types pommés des types fourragers (fig 1B).

Chaque population est par ailleurs distincte et relativement homogène dans le premier plan de l'analyse pour les marqueurs RAPD alors que les mêmes populations sont non distinctes et plus hétérogènes par l'analyse isoenzymatique. La forte variabilité intrapopulation révélée au niveau enzymatique masque probablement une variabilité interpopulation et intercultigroupe, qui est en revanche bien révélée au niveau des marqueurs RAPDs.

\section{Construction d'arbres phylogénétiques}

Les 2 arbres phylogénétiques obtenus à partir de 26 allèles isoenzymatiques (fig $2 \mathrm{~A}$ ) et 101 marqueurs RAPDs (fig 2B) présentent de nombreuses similitudes.

La première dichotomie sépare dans les 2 cas les populations de types choux-fleurs des autres populations. À l'intérieur de ce cultigroupe, l'analyse isoenzymatique distingue les populations de précocité décembre à mars (DJ 105b, FLH 22.01 et FLH 22.07) des populations plus tardives (mai à août). L'analyse RAPD quant à elle permet de différencier les types été (FLE \#), des types hiver (FLH, M-71 et DJ 105b).

Les autres populations forment 3 groupes pour l'analyse isoenzymatique, qui correspondent pour l'un aux populations de choux-fourragers et pour les 2 autres aux populations de choux pommés. L'un de ces 2 derniers groupes ne contient que le type cabus tandis que l'autre contient des types Milan et cabus. Pour l'analyse isoenzymatique, le groupe de choux fourragers est structuré sans lien apparent avec les données agronomiques recueillies. 


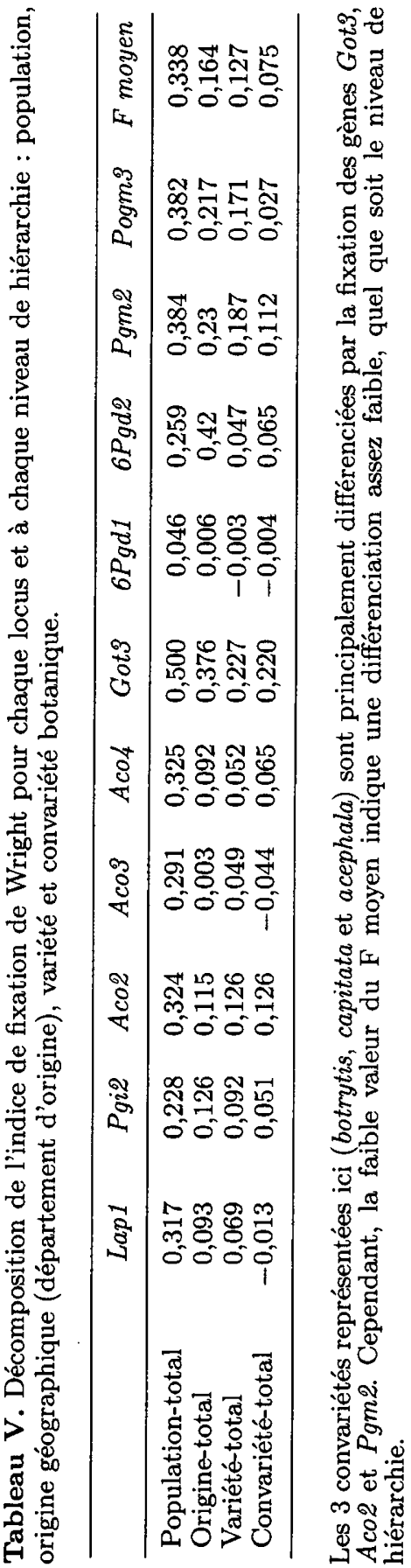


Tableau VI. Matrice des distances de Rogers (1972) entre les convariétés à partir de données isoenzymatiques (A) et RAPD (B).

\begin{tabular}{lcccc}
\hline Convariété & Nb de population & botrytis & capitata & acephala \\
\hline A & & & & \\
botrytis & 8 & 0,184 & & \\
capitata & 9 & 0,337 & 0,273 & 0,248 \\
acephala & 11 & 0,37 & 0,317 & \\
B & & & & \\
botrytis & 8 & 0,222 & & \\
capitata & 9 & 0,549 & 0,249 & 0,28 \\
acephala & 11 & 0,494 & 0,53 & \\
\hline
\end{tabular}

Pour l'analyse RAPD la distinction est nette entre les types fourragers et pommés. Ces derniers se séparent en types Milan et cabus conformément aux caractéristiques botaniques. Les résultats obtenus pour le groupe de choux fourragers apparaissent cohérents avec ceux obtenus sur la base des données morphoagronomiques, contrairement à ce qui est observé pour les isozymes. Le type cavalier rouge (Flamcaul) se distingue par son originalité puis les types demi-moelliers et moelliers (var medullosa) semblent diverger de manière homogène, excepté pour la population FO22-47.

Une différence majeure apparaît dans la distribution de la variabilité génétique pour ces 2 types de marqueurs. En effet, très peu d'allèles isoenzymatiques sont fixés et caractéristiques d'un cultigroupe. En revanche, de nombreux marqueurs RAPDs sont présents spécifiquement au sein des cultigroupes et des groupes morphologiques. La distance moyenne de Rogers (1972) intracultigroupes est peu différente pour les 2 types de marqueurs. En revanche, les distances inter-cultigroupes sont beaucoup plus importantes pour les RAPDs (tableau VI) : le rapport moyen des distances intra- et intercultigroupe est de 0,689 pour les isoenzymes, alors qu'il n'est que de 0,477 pour les RAPDs.

\section{DISCUSSION}

L'objectif de cette étude était de comparer 2 outils d'analyse de la diversité génétique (isozymes et RAPDs) pour leur efficacité à structurer la collection de choux étudiée. Les résultats ont donc été discutés en se référant aux données morpho-agronomiques disponibles sur ces populations.

\section{La variabilité génétique intrapopulation}

La variabilité génétique appréciée à partir des données isoenzymatiques donne des valeurs de l'indice de Nei corrélées avec le régime de reproduction des populations. Pour les autogames strictes (choux-fleurs d'été), la variabilité intrapopulation est nulle ou quasi nulle. Pour les allogames préférentielles les indices sont comparables et même supérieurs à ceux observés chez le maïs (Lavergne, 1988). Cette variabilité 

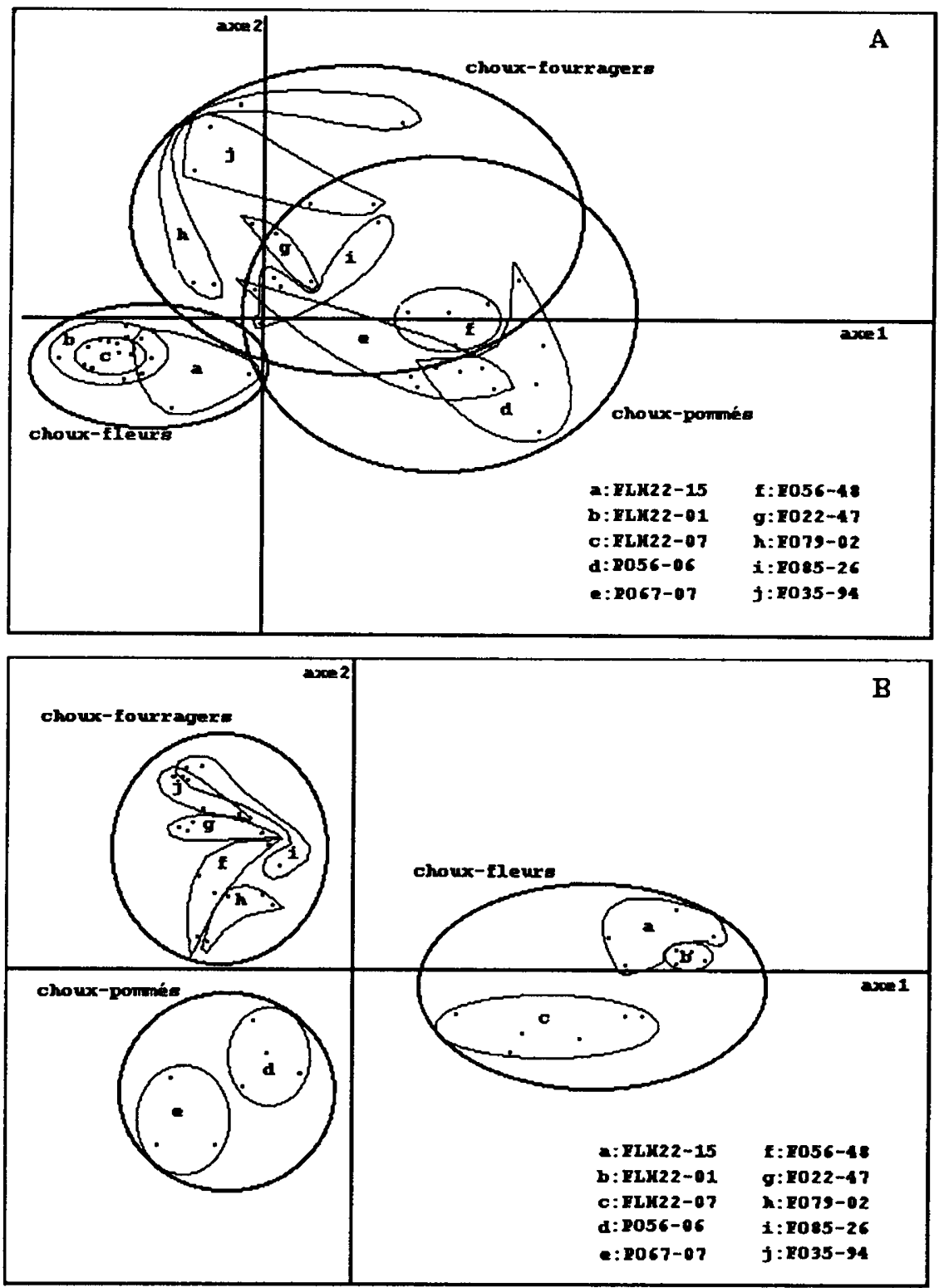

Fig 1. AFCM (analyse factorielle des correspondances multiples) réalisée à partir de la présence-absence de 28 allèles isoenzymatiques (A) et 28 marqueurs RAPDs (B). Chaque point représente un individu et chaque sous-groupe une population. Les grands ensembles représentent les cultigroupes. Il apparaît clairement que chaque cultigroupe et chaque population est plus homogène et distinct lorsqu'il est défini à l'aide de données RAPDs. En revanche, la faible différenciation interpopulations et intercultigroupes obtenue par l'analyse isoenzymatique est un obstacle à la structuration de la variabilité génétique. 


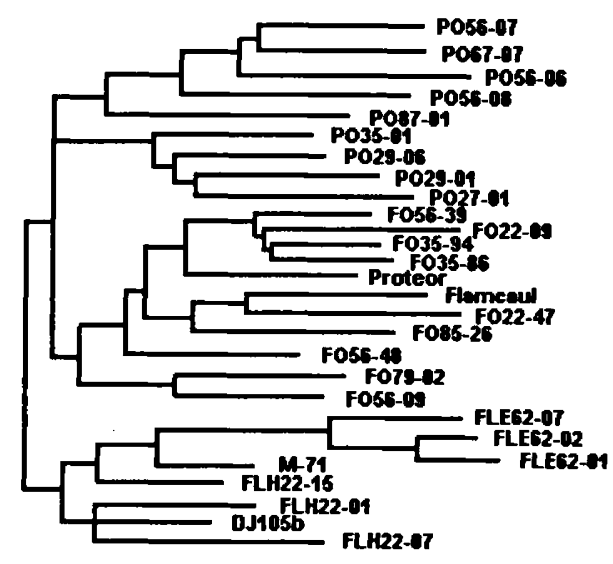

A

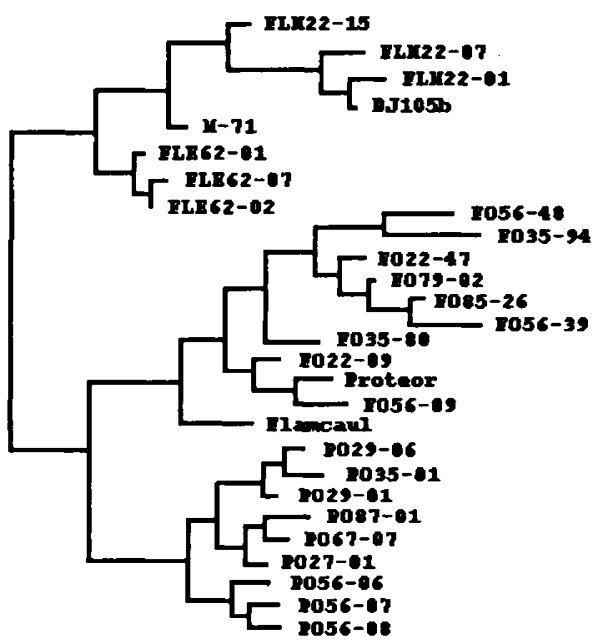

B

Fig 2. Arbres phylogénétiques réalisés par la méthode de groupage UPGMA (unweighted pair-group method with arithmetic averaging). A. Hiérarchisation obtenue par l'analyse de la fréquence de 26 allèles appartenant à 10 gènes isoenzymatiques sur 30 plantes par population. B. Hiérarchisation obtenue par l'analyse de 101 marqueurs RAPDs sur un échantillon d'ADN de 40 graines en mélange. On obtient par l'analyse moléculaire une bonne ségrégation des groupes morphologiques, en plein accord avec les données phylogénétiques connues.

génétique s'exprime par la présence dans de nombreuses populations de la presque totalité des allèles recensés dans la collection. Cette forte variabilité intrapopulation est un obstacle à la structuration.

L'analyse factorielle des correspondances multiples, réalisée sur la base des données isoenzymatiques de présence-absence de bande, confirme l'importance de la diversité intrapopulation dans la diversité totale. La même analyse, réalisée sur la base des données RAPDs, conduit à une différentiation plus nette des populations. Il semble que le rapport variabilité intrapopulation sur inter-population soit plus faible pour les RAPDs que pour les isoenzymes. Les observations n'ont pas donné lieu à des mesures chiffrées car l'effectif dans chaque population est trop faible. Cependant, le fait d'observer le même phénomène pour toutes les populations nous conduit à penser que les marqueurs RAPDs fournissent des informations plus spécifiques aux populations que ne le font les informations isoenzymatiques. Cela nous a permis de caractériser simplement chaque population avec un seul échantillon (provenant du mélange de 40 graines), et en faisant volontairement abstraction de la variabilité intrapopulation. 


\section{La variabilité génétique interpopulations}

Au niveau inter-populations, la structuration observée sur la base des données RAPDs est plus cohérente avec celle obtenue pour leurs caractéristiques agronomiques, que celle observée sur la base des données isoenzymatiques.

Pour les choux-fleurs, on observe une bonne représentation phylogénétique en accord avec la précocité. Il est intéressant de noter que la distinction se fait à partir du régime de reproduction pour l'analyse isoenzymatique : les types allogames (décembre à mars) et les types autogames (mai à septembre) sont regroupés dans des ensembles différents. Pour l'analyse RAPD, les regroupements correspondent aux adaptations écogéographiques : les types hiver (décembre à mai) cultivés en Bretagne, et les types été (juillet à septembre) cultivés dans le nord de la France et dérivés d'un pool génétique hiver tardif (Hervé, communication personnelle).

Pour les populations de choux pommés, la séparation en 2 groupes ne correspond pas clairement aux données morphologiques et botaniques. On distingue en effet le type Milan à feuilles frisées (sabauda) et le type cabus à feuilles lisses (capitata). Si cette distinction est clairement observée dans l'analyse des RAPDs, elle ne l'est pas totalement pour l'analyse des isozymes. En outre, si toutes les populations de choux pommés présentent des caractéristiques communes et propres sur la base des RAPDs, elles sont mélangées aux populations de choux fourragers, sur la base des données isoenzymatiques.

Pour la structuration des choux fourragers, aucune corrélation avec des données agronomiques n'est observée pour l'analyse des isozymes. En revanche certains groupes homogènes sont observés par l'analyse RAPD (demi-moellier, moellier). Ce cultigroupe est probablement le plus difficile à structurer car les méthodes de sélection des cultivars ont probablement favorisé les mélanges de variétés. Par ailleurs, la multiplicité des types morphologiques et la subjectivité de leurs caractéristiques (différence entre le type demi-moellier et moellier) relativise l'authenticité des informations contenues dans le répertoire initial.

\section{Les caractéristiques des marqueurs employés}

Dans cette étude comparative, le nombre et la nature des caractères étudiés sont fort différents. Pour l'analyse isoenzymatique, 30 plantes ont été étudiées pour 10 gènes, soit 600 informations alléliques recueillies pour chaque population. Dans l'analyse RAPD, 101 locus ont été utilisés pour définir chaque population. La nature des caractères est également différente puisqu'il s'agit de marqueurs codominants dans le cas des isoenzymes, et de marqueurs dominants dans celui des RAPDs. D'autre part, les marqueurs isoenzymatiques sont un produit de l'ADN exprimé, tandis que les marqueurs RAPDs sont obtenus à partir de l'ADN total.

Par ailleurs, les marqueurs isoenzymatiques et RAPDs sont des caractères pour lesquels l'évolution est différente car soumise à des lois particulières. Seule une petite partie des mutations sur l'ADN des gènes isoenzymatiques est révélée par électrophorèse (Brown et Weir, 1983), tandis que la plupart des mutations est révélée par RAPD. De plus, cet $\mathrm{ADN}$ amplifié est pour la plupart non codant et à ce titre vérifie complètement la théorie neutraliste. Les gènes isoenzymatiques quant à eux codent pour des protéines et sont soumis aux règles de la fonctionnalité. 
Il est généralement admis que plus une molécule est fonctionnellement active, plus sa vitesse d'évolution est faible (Kimura, 1991). Ainsi, le temps d'évolution qui a séparé les choux-fleurs des autres choux semble suffisamment important pour que des caractères isoenzymatiques spécifiques puissent être sélectionnés par ce que Kimura désigne par «dérive génétique aléatoire» (1980). En revanche, à l'intérieur des cultigroupes des choux pommés et fourragers, l'évolution lente des isoenzymes n'aurait pas permis leur nette distinction et leur structuration, contrairement aux marqueurs RAPDs.

Ces 2 sources d'informations sont donc différentes et complémentaires. Pour l'analyse de la variabilité intrapopulation, l'utilisation de marqueurs RAPDs est difficile et coûteuse à développer plante à plante. Cette technique est valorisée économiquement si elle est appliquée sur un échantillon représentatif de la population. Par ailleurs, la nature codominante des caractères isoenzymatiques peut être exploitée en particulier dans l'étude de l'évolution des populations et des pools génétiques, ou des conditions de multiplication (effectif minimal, dérive génétique, migration). L' objectif étant ici la constitution de pools de populations homogènes, une technique rapide, peu coûteuse et informative, était recherchée pour se substituer à l'évaluation phénotypique des populations. En ce qui concerne la structuration des populations dans la collection, la méthode RAPD s'est révélée correspondre le mieux aux données phylogéniques et agronomiques connues. Cette méthode pourrait être optimisée en choisissant une vingtaine d'amorces informatives, c'est-à-dire révélant un nombre important de marqueurs impliqués dans la structuration des populations à l'intérieur des cultigroupes. En effet, l'appartenance au cultigroupe étant une information connue pour chaque population, il est judicieux de structurer ces cultigroupes séparément. Seuls les marqueurs permettant de différencier et de structurer les populations à l'intérieur d'un groupe cultivé seront retenus (les autres bandes étant monomorphes et caractéristiques du cultigroupe). Ce type d'analyse, réalisé sur chacun des 3 cultigroupes, a aussi donné des résultats en accord avec les données morpho-agronomiques (Margalé, soumis).

La comparaison entre les informations apportées sur la structuration de la diversité génétique des populations de choux et le coût financier de l'opération est à l'avantage des marqueurs RAPDs utilisés selon le protocole présenté sur un bulk de graines. Cela vaut pour des allogames mais à plus forte raison pour des autogames puisque nous négligeons par cette méthode la variabilité intrapopulation. Cet avantage est associé à l'infinité de marqueurs disponibles par l'analyse RAPD, mais aussi à l'évolution différente des 2 types de caractères. La méthode RAPD est tout particulièrement recommandée lorsque l'on cherche à structurer une collection de populations ayant peu divergé. Ces méthodes moléculaires permettent de constituer des pools génétiques à moindre coût mais il est nécessaire par la suite de caractériser ces derniers à l'aide de critères morpho-agronomiques.

\section{RÉFÉRENCES}

Arus P, Shields CR (1983) Cole crops. In: Isozymes in plant genetics and breeding. Part B (Tanksley SD, Orton TJ, eds). Elsevier, Amsterdam, 339-350

Arus P, Chevre AM, Delourme R, Eber F, Kerlan MC, Margalé E, Quiros CF (1991) Isozyme nomenclature for eight isozyme systems in three Brassica species. 
In: Proceedings of the 8th International Rapeseed Congress. Saskatoon, 9-11 juillet 1991, 1061-1066

Brown AHD, Weir BS (1983) Measuring genetic variability in plant populations. In: Isozymes in plant genetics and breeding. Part A (Tanksley SD, Orton TJ, eds). Elsevier, Amsterdam, 219-240

Dellaporta SL, Wood J, Hicks JB (1983) A plant molecular DNA minipreparation version: II. Plant Mol Biol Rep 1, 19-21

Fitch WM, Margoliash E (1967) Construction of phylogenetic trees. Science 155, 279-284

Crisp P (1975) Trends in the breeding and cultivation of cruciferous crops. In: The biology and chemistry of the Cruciferae (Vaughan et al, eds). London, 69-118

Kimura M (1980) La théorie neutraliste de l'évolution moléculaire. Pour Sci 27, 48-56

Kimura M (1991) The neutral theory of molecular evolution: A review of recent evidence. Jpn J Genet 66, 367-386

Lavergne V (1988) Variabilité entre populations de maïs (Zea mays L). Approche biométrique et approche enzymatique. Thèse de doctorat INAPG, Paris

Lefort-Buson M, de Vienne D (1985) Les distances génétiques : estimations et applications. INRA, Paris

Prakash S, Hinata K (1980) Taxonomy, cytogenetics and origin of crop Brassicas, a review. Opera Bot $55,1-57$

Rogers JS (1972) Measures of genetic similarity and genetic distances. In: Studies Genet VII 7213, 145-153

Williams JGK, Kubelil AE, Levak KJ, Rafalski JA, Tingey SC (1990) DNA polymorphisms amplified by arbitrary primers are useful as genetic markers. Nucleic Acids Res 18, 6531-6535 